\title{
CORRELATION ANALYSIS OF ORDERED SYMMETRICALLY DEPENDENT OBSERVATIONS AND THEIR CONCOMITANTS OF ORDER STATISTICS
}

\author{
MARLOS VIANA AND HAK-MYUNG LEE
}

\begin{abstract}
Given two jointly observed random vectors $\mathbf{Y}$ and $\mathbf{Z}$ of same dimension, we consider the ordered version $\mathcal{Y}$ of $\mathbf{Y}$ and the resulting induced or concomitant of order statistics $\mathcal{Z}$. When $\mathrm{X}$ is a covariate of interest, also jointly observed with $\mathbf{Y}$ and $\mathbf{Z}$, and assuming that the joint covariance structure of $(X, \mathbf{Y}, \mathbf{Z})$ is permutation-symmetric, the joint covariance structure of $(X, \mathcal{Y}, \mathcal{Z})$ and related correlation parameters are explicitly obtained when the joint probability distribution of $(X, \mathbf{Y}, \mathbf{Z})$ is normal. Extensions to elliptically contoured distributions are also discussed.
\end{abstract}

\section{INTRODUCTION}

The present paper describes the covariance structure and associated correlation parameters among ordered observations, their concomitants of order statistics and one or more covariates. In each experimental unit, the vectors $\mathbf{Y}$ and $\mathbf{Z}$ are observed, in addition to a covariate, $\mathbf{X}$. The $\mathbf{Y}$ vector is ordered according to a given rule, and the resulting vector indicated by $\mathcal{Y}$. This induces the concomitant order, $\mathcal{Z}$, in $\mathbf{Z}$. Our paper is focused on the covariance structure of $(X, \mathcal{Y}, \mathcal{Z})$. Our work is motivated by experimental conditions in which there is a natural dependence structure among the components of $\mathbf{Y}$, among the components of $\mathbf{Z}$, and among $\mathbf{X}, \mathbf{Y}$ and $\mathbf{Z}$. For example, in vision research, extreme observations between fellow eyes are often used to describe visual acuity. Normally, a joint pair of measures $Y_{1}, Y_{2}$ is observed in each subject, representing the visual acuity of fellow eyes. In addition, another pair of measures $\left(Z_{1}, Z_{2}\right)$, representing, say, the intra-ocular pressure (IOP) of fellow eyes, is observed. Often, one or more covariates, are also of interest (e.g., the subject's age). Because visual acuity responses generally are unequal, of interest are not the initial measures $Y_{1}, Y_{2}$ but rather the extreme acuity responses, the best acuity $Y_{(1)}$ and the worst acuity $Y_{(2)}$. The resulting IOP in the eye with best acuity is a concomitant of order statistic, and so is the IOP in

1991 Mathematics Subject Classification. 62G30, 62J05.

Key words and phrases. order statistics, concomitants, permutation-symmetry.

Supported in part by an unrestricted grant from Research to Prevent Blindness, NY, to the Department of Ophthalmology and Visual Sciences. We are indebted to C. Olopade, MD, UIC Sleep Center and Clinical Research Center for the access to his pilot data. The authors are thankful to two referees for their judicious comments and suggestions. To appear, subject to editorial changes, in The Canadian Journal of Statistics. Revised May 9, 2005. 
the eye with worst acuity. Consequently, there is interest in making inferences on the covariance structure of $(X, \mathcal{Y}, \mathcal{Z})$. Earlier developments of these applications may be seen in Olkin and Viana (1995) (see also David (1996)) , Viana and Olkin (1997), Viana (1998) and Lee and Viana (1999). Also of related interest is Viana, Olkin and McMahon (1993). In what follows we introduce some of the notation and assumptions of dependence among the joint components of $\mathbf{Y}, \mathbf{Z}$ and X. In Section 2 the joint covariance structure of $(X, \mathcal{Y}, \mathcal{Z})$ is obtained followed by the correlation parameters in Section 3. A numerical example is included in Section 4, followed by complementary results and related approaches in Section 5.

Let $\mathbf{Y}$ and $\mathbf{Z}$ indicate jointly observed random vectors in $R^{p}$ with covariance structure defined by

$$
\begin{aligned}
& \Sigma_{11}=\operatorname{Cov}(\mathbf{Y})=\sigma_{1}^{2}\left(\gamma_{11} \mathbf{e e}^{\prime}+\left(1-\gamma_{11}\right) \mathbf{I}\right), \\
& \Sigma_{22}=\operatorname{Cov}(\mathbf{Z})=\sigma_{2}^{2}\left(\gamma_{22} \mathbf{e e}^{\prime}+\left(1-\gamma_{22}\right) \mathbf{I}\right), \\
& \Sigma_{12}=\operatorname{Cov}(\mathbf{Y}, \mathbf{Z})=\sigma_{1} \sigma_{2}\left(\gamma_{12} \mathbf{e e}^{\prime}+\left(\lambda_{12}-\gamma_{12}\right) \mathbf{I}\right),
\end{aligned}
$$

where $\mathbf{e}^{\prime}=(1, \ldots, 1)$ has $p$ components and $\mathbf{I}$ is the identity matrix of corresponding dimension. In addition, let $\mathrm{X}$ indicate a real-valued random variable, jointly observed with $\mathbf{Y}$ and $\mathbf{Z}$, with covariance structure

$$
\Sigma_{00}=\sigma_{0}^{2}, \quad \Sigma_{01}=\operatorname{Cov}(X, \mathbf{Y})=\sigma_{0} \sigma_{1} \gamma_{01} \mathbf{e}^{\prime}, \quad \Sigma_{02}=\operatorname{Cov}(X, \mathbf{Z})=\sigma_{0} \sigma_{2} \gamma_{02} \mathbf{e}^{\prime}
$$

When the block components $\Sigma_{i j}$ of $\Sigma=\operatorname{Cov}[(X, \mathbf{Y}, \mathbf{Z})]$ satisfy these symmetry conditions, we say that $\Sigma$ is block-permutation symmetric (BPS ), or simply, that $\Sigma$ is BPS. Similarly, we say that the vector of means $\boldsymbol{\mu}=\left(\mu_{0}, \boldsymbol{\mu}_{1}, \boldsymbol{\mu}_{2}\right)$ is BPS when $\boldsymbol{\mu}_{i}=\mu_{i} \mathbf{e}$, for scalars $\mu_{i}, \mathrm{i}=1,2$. We remark that $\operatorname{Cov}[(X, \mathbf{Y}, \mathbf{Z})]$ indicates the block-covariance matrix $\Sigma$, whereas, for example, $\operatorname{Cov}(\mathbf{Y}, \mathbf{Z})$ denotes the cross-covariance block $\Sigma_{12}$ of $\Sigma$. If $F$ indicates the joint probability distribution of $(\mathbf{Y}, \mathbf{Z})$, and $F(g \mathbf{y}, g \mathbf{z})=F(\mathbf{y}, \mathbf{z})$ for all $p \times p$ permutation matrices $g$, we say that $F$ has the BPS property. In this case, it follows that, for all $g$, $g \boldsymbol{\mu}_{i}=\boldsymbol{\mu}_{i}, g \Sigma_{i j} g^{\prime}=\Sigma_{i j}, i, j=1,2$. This implies that both $\boldsymbol{\mu}$ and $\Sigma$ satisfy the BPS conditions. Throughout this paper, unless otherwise indicated, it is assumed that the underlying joint distribution of $(X, \mathbf{Y}, \mathbf{Z})$ has the BPS property.

We indicate by $\mathcal{Y}^{\prime}=\left(Y_{(1)}, Y_{(2)}, \ldots, Y_{(p)}\right)$ the ordered version of $\mathbf{Y}$ according to $Y_{(1)} \leq \ldots \leq Y_{(p)}$. Correspondingly, $\mathcal{Z}$ indicates the vector of induced or concomitant order statistics. The component $Z_{[i]}$ of $\mathcal{Z}$ is defined by $Z_{[i]}=Z_{j}$ whenever $Y_{(i)}=Y_{j}$. Then, $\mathcal{Z}$ is well-defined, provided that the underlying probability model of $\mathbf{Y}$ assigns probability zero to ties.

The standard formulation of concomitants is usually defined for $N$ independent (e.g., a sample) observations from the underlying probability model of a bivariate vector with covariance structure $\Psi$. In that formulation, $\mathcal{Y}$ indicates the ordered sample and $\mathcal{Z}$ the concomitants. The resulting covariance structure $\Sigma$ may be expressed in block form with $\Sigma_{12}=\gamma \sigma_{1} \sigma_{2} \mathbf{I}$ and $\Sigma_{i i}=\sigma_{i}^{2} \mathbf{I}, i=1,2$, where $\sigma_{1}^{2}, \sigma_{2}^{2}$ and $\gamma$ are the parameters defining the bivariate structure in $\Psi$. The standard theory has its roots in David and Galambos 
(1974) and Bhattacharya $(1976,1984)$. A comprehensive, more recent, account of developments in the area is found in David and Nagaraja (1998).

\section{The Joint Covariance structure of $(X, \mathcal{Y}, \mathcal{Z})$}

2.1. The joint covariance structure of $(\mathcal{Y}, \mathcal{Z})$. Assume that $\mathcal{Z}$, the vector of induced order statistics, is well-defined. We start with the following remark: If $\mathbf{Y}$ and $\mathbf{Z}$ are jointly independent, then $\mathcal{Y}$ and $\mathcal{Z}$ are jointly independent and $\mathbf{Z} \equiv \mathcal{Z}$ in distribution. $\quad$ In fact, if $(\mathbf{Y}, \mathbf{Z})$ is $\mathrm{BPS}$, then, marginally, $\mathbf{Y}$ and $\mathbf{Z}$ are permutation symmetric and the distribution of $\mathcal{Z}$ can be represented by the distribution of $\mathbf{U Z}$ where $\mathbf{U}$ is a random permutation matrix representing the permutation of $\{1,2, \ldots, p\}$ generated by ranking the components of $\mathbf{Y}$. Moreover, $\mathbf{U}$ is uniform in its class, depends on $\mathbf{Y}$ alone and hence is independent of $\mathcal{Z}$. This implies that $\mathcal{Z} \equiv \mathbf{U Z} \equiv \mathbf{Z}$ in distribution. It also implies that $\mathcal{Y}$ and $\mathcal{Z}$ are jointly independent. The representation UZ is discussed in more detail in Section 5.4. The following proposition is based on the above remark and is proved in Lee and Viana (1999):

Proposition 2.1. Suppose that $(\mathbf{Y}, \mathbf{Z})$ is BPS and that $\mathbf{Z}=\mathbf{T Y}+\mathbf{V}$, where $\mathbf{V}$ and $\mathbf{Y}$ are jointly independent and $\mathbf{T}$ is a permutation symmetric constant matrix (and hence $\mathbf{T}=\mathbf{T}^{\prime}$ ). Then

$$
\operatorname{Cov}[(\mathcal{Y}, \mathcal{Z})]=\left[\begin{array}{ll}
\operatorname{Cov}(\mathcal{Y}) & \operatorname{Cov}(\mathcal{Y}) \mathbf{T} \\
\mathbf{T C o v}(\mathcal{Y}) & \Sigma_{22}+\mathbf{T}\left(\operatorname{Cov}(\mathcal{Y})-\Sigma_{11}\right) \mathbf{T}
\end{array}\right]
$$

Under these conditions, note that $E(\mathcal{Z})=\boldsymbol{\mu}_{2} \mathbf{e}+\mathbf{T}\left(E(\mathcal{Y})-\boldsymbol{\mu}_{1}\right)$, which follows directly from $\mathcal{Z}=\mathbf{T} \mathcal{Y}+\mathcal{V}$ and $E(\mathbf{V})=\boldsymbol{\mu}_{2}-\mathbf{T} \boldsymbol{\mu}_{1}$.

Corollary 2.1. If the joint distribution of $(\mathbf{Y}, \mathbf{Z})$ is multivariate normal satisfying the BPS condition, then

$$
\operatorname{Cov}[(\mathcal{Y}, \mathcal{Z})]=\left[\begin{array}{ll}
\Sigma_{11} \mathcal{C} & \Sigma_{12} \mathcal{C} \\
\Sigma_{21} \mathcal{C} & \Sigma_{22}+\frac{\sigma_{2}^{2}\left(\lambda_{12}-\gamma_{12}\right)^{2}}{\left(1-\gamma_{11}\right)}(\mathcal{C}-\mathbf{I})
\end{array}\right]
$$

where $\mathcal{C}$ is the covariance matrix of $p$ ordered independent standard normal variates.

Proof. In Proposition 2.1, let $\mathbf{T}=\Sigma_{21} \Sigma_{11}^{-1}$ so that $\mathbf{T}$ is permutation symmetric, $\operatorname{Cov}(\mathbf{Z}-\mathbf{T Y}, \mathbf{Y})=0$ and hence, $\mathbf{V}=\mathbf{Z}-\mathbf{T Y}$ and $\mathbf{Y}$ are jointly independent. In addition, from Olkin and Viana (1995), we have $\operatorname{Cov}(\mathcal{Y})=\Sigma_{11} \mathcal{C}$. To evaluate $\operatorname{Cov}(\mathcal{Z})$, we observe that $\mathbf{e}^{\prime}(\mathcal{C}-\mathbf{I})=0$, and hence

$$
T\left(\operatorname{Cov}(\mathcal{Y})-\Sigma_{11}\right) T=T\left(\Sigma_{11} \mathcal{C}-\Sigma_{11}\right) T=T \Sigma_{11} T(\mathcal{C}-\mathbf{I})=\Sigma_{21}^{2} \Sigma_{11}^{-1}(\mathcal{C}-\mathbf{I})=\frac{\sigma_{2}^{2}\left(\lambda_{12}-\gamma_{12}\right)^{2}}{\left(1-\gamma_{11}\right)}(\mathcal{C}-\mathbf{I})
$$

completing the proof.

It also follows from Corollary 2.1 that $E(\mathcal{Z})=\boldsymbol{\mu}_{2}+\frac{\sigma_{2}\left(\lambda_{12}-\gamma_{12}\right)}{\sqrt{1-\gamma_{11}}} \mathbf{c}$, where $\mathbf{c}$ is the mean vector of $p$ ordered independent standard normal variates (use the fact, e.g., Owen and Steck $(1962)$, that $E(\mathcal{Y})=$ $\left.\boldsymbol{\mu}_{1}+\sigma_{1} \sqrt{1-\gamma_{11}} \mathbf{c}\right)$ 
2.2. The joint covariance structure of $(X, \mathcal{Y})$. Suppose that $\mathbf{W}^{\prime}=(X, \mathbf{Y})$ has a $(p+1)$-dimensional elliptically contoured (EC) distribution and $\operatorname{Cov}\left(X, Y_{i}\right)=\operatorname{Cov}\left(X, Y_{j}\right), i, j=1, \ldots, p$. Its density function f has the form $f(\mathbf{w})=|\Sigma|^{-1 / 2} g\left((\mathbf{w}-\boldsymbol{\mu})^{\prime} \Sigma^{-1}(\mathbf{w}-\boldsymbol{\mu})\right)$ for non increasing function $g, \Sigma$ positive definite, $\boldsymbol{\mu}^{\prime}=\left(\mu_{0}, \mu_{1}, \ldots, \mu_{p}\right)$ and

$$
\Sigma=\left[\begin{array}{cc}
\sigma_{0}^{2} & \alpha \mathbf{e}^{\prime} \\
\alpha \mathbf{e} & \Sigma_{11}
\end{array}\right] .
$$

Let $\mathbf{Y}_{\delta}^{\prime}=\left(Y_{1}-Y_{p}, \ldots, Y_{p-1}-Y_{p}\right)$ indicate the random spacings with respect to, say, $Y_{p}$ (as it will turn out, the choice of the particular component is irrelevant). We need the following Lemma, proved in Appendix A.

Lemma 2.1. If $(X, \mathbf{Y})$ has $(p+1)$-dimensional $\mathbf{E C}$ distribution and $\operatorname{Cov}\left(X, Y_{i}\right)=\operatorname{Cov}\left(X, Y_{j}\right), i, j=1, \ldots, p$, then, the marginal joint distribution of $\left(X, \mathbf{Y}_{\delta}\right)$ is an even function of $X-\mu_{0}$.

When restricted to the multivariate normal distribution, Lemma 2.1 says that $X$ and $\mathbf{Y}_{\delta}$ are independent when $\operatorname{Cov}\left(X, Y_{i}\right)=\operatorname{Cov}\left(X, Y_{j}\right)$, in which case,

$$
\operatorname{Cov}\left[\begin{array}{l}
X \\
\mathbf{Y}_{\delta}
\end{array}\right]=\left[\begin{array}{cc}
\sigma_{0}^{2} & \mathbf{0} \\
\mathbf{0} & \Sigma_{\delta}
\end{array}\right],
$$

where the components $\sigma_{i j}$ of $\Sigma_{\delta}$ are given by $\sigma_{i}^{2}-2 \sigma_{i p}+\sigma_{p}^{2}$, when $i=j$, and by $\sigma_{i j}-\sigma_{i p}-\sigma_{j p}+\sigma_{p}^{2}$ when $i \neq j$. The distribution of $\mathbf{Y}_{\delta}$ is $\mathrm{N}\left(\boldsymbol{\mu}_{\delta}, \Sigma_{\delta}\right)$. The following proposition is an extension of Theorem 1 in Olkin and Viana (1995). We provide an outline of the proof, which is similar to the former result.

Proposition 2.2. If the distribution of $X, \mathbf{Y}$ is nondegenerate $\mathbf{E C}$ subject to $\operatorname{Cov}\left(X, Y_{i}\right)=\operatorname{Cov}\left(X, Y_{j}\right)$, $i, j=1, \ldots, p$, then $\operatorname{Cov}(X, \mathcal{Y})=\operatorname{Cov}(X, \mathbf{Y})$.

Proof. Fix $1 \leq r \leq p$ and let $E_{j}$ denote the event $\left\{Y_{j}=Y_{(r)}\right\}, j=1, \ldots, p$, so that $\operatorname{Cov}\left(X, Y_{(r)}\right)=$ $\sum_{j=1}^{p} \operatorname{Cov}\left(X, Y_{j} \mid E_{j}\right) P_{Y}\left(E_{j}\right)$ and $\operatorname{Cov}\left(X, Y_{r}\right)=\sum_{j=1}^{p} \operatorname{Cov}\left(X, Y_{r} \mid E_{j}\right) P_{Y}\left(E_{j}\right)$. To show $\operatorname{Cov}\left(X, Y_{(r)}\right)=\operatorname{Cov}\left(X, Y_{r}\right)$, it is sufficient to show $\operatorname{Cov}\left(X, Y_{r}-Y_{j} \mid E_{j}\right)=0, j=1, \ldots, p$. Take $j=p$, say, and let $f$ indicate the joint density function of $x, \mathbf{y}$. Then,

$$
\operatorname{Cov}\left(X, Y_{r}-Y_{p} \mid E_{p}\right)=\frac{1}{P\left(E_{p}\right)} \int_{X \times E_{p}}\left(x-\mu_{0}\right)\left(\left(y_{r}-\mu_{r}\right)-\left(y_{p}-\mu_{p}\right)\right) f(x, \mathbf{y}) d x d \mathbf{y} .
$$

The event $\mathbf{Y} \in E_{p}$ is invariant under the linear transformation $(X, \mathbf{Y}) \rightarrow V=\left(X, \mathbf{Y}_{\delta}, Y_{p}\right)$, so that

$$
\operatorname{Cov}\left(X, Y_{r}-Y_{p} \mid E_{p}\right)=\frac{1}{P\left(E_{p}\right)} \int_{X \times E_{p}}\left(x-\mu_{0}\right)\left(\mathbf{y}_{\delta r}-\mu_{\delta r}\right) f\left(x, \mathbf{y}_{\delta}\right) d x d \mathbf{y}_{\delta}
$$

where $y_{\delta r}=y_{r}-y_{p}$ and $\mu_{\delta r}=\mu_{r}-\mu_{p}$. However, because $f\left(x, \mathbf{y}_{\delta}\right)$ is an even function of $\left(x-\mu_{0}\right)$, the integral of $X$ over the real line is zero, and consequently $\operatorname{Cov}\left(X, Y_{r}-Y_{p} \mid E_{p}\right)=0$. This holds for an arbitrary choice of $\mathrm{j}$. 
If $(X, \mathbf{Y})$ is multivariate normal, the corresponding result has a more explicit formulation. Because $\mathbf{Y}_{\delta}$ is independent of $X$, as discussed earlier, the integral in expression (2.4) becomes

$$
\int_{\mathbf{X}}\left(x-\mu_{0}\right) f(x)\left[\int_{E_{p}}\left(\mathbf{y}_{\delta r}-\mu_{\delta r}\right) f\left(\mathbf{y}_{\delta}\right) d \mathbf{y}_{\delta}\right] d x \text {. }
$$

The inside integral can be factored out so that the whole integral will be zero. In particular, then, if the distribution of $(X, \mathbf{Y})$ is multivariate normal and $\operatorname{Cov}\left(X, Y_{i}\right)=\operatorname{Cov}\left(X, Y_{j}\right), i, j=1, \ldots, p, \operatorname{then} \operatorname{Cov}(X, \mathcal{Y})=$ $\operatorname{Cov}(X, \mathbf{Y})$.

2.3. The joint covariance structure of $(X, \mathcal{Z})$. To extend Proposition 2.1 to $(X, \mathbf{Y}, \mathbf{Z})$, suppose that $(X, \mathbf{Y}, \mathbf{Z})$ has $(2 k+1)$-dimensional EC distribution and define, in analogy,

$$
\mathbf{W}^{\prime}=\left(X, Y_{1}, \ldots, Y_{p-1}, Z_{1}, \ldots, Z_{p-1}, Y_{p}, Z_{p}\right)
$$

so that its distribution is $\mathbf{E C}(\boldsymbol{\mu}, \Sigma)$ with $\boldsymbol{\mu}^{\prime}=\left(\mu_{0}, \mu_{11}, \ldots, \mu_{1(p-1)}, \mu_{21}, \ldots, \mu_{1(p-1)}, \mu_{1 p}, \mu_{2 p}\right)$,

$$
\Sigma=\left[\begin{array}{ccc}
\sigma_{0}^{2} & \left(\alpha \mathbf{e}^{\prime}, \beta \mathbf{e}^{\prime}\right) & (\alpha, \beta) \\
& \Sigma_{1} & \Sigma_{2} \\
& & \sigma_{p}^{2}
\end{array}\right] \text {, and } \sigma_{p}^{2}=\left[\begin{array}{cc}
\sigma_{1 p}^{2} & \sigma_{12 p} \\
\sigma_{12 p} & \sigma_{2 p}^{2}
\end{array}\right]
$$

In addition, let $\mathbf{Y}_{\delta}$ indicate the random spacing with respect to $Y_{p}$ in $\mathbf{Y}$ and $\mathbf{Z}_{\delta}$ the random spacing with respect to $Z_{p}$ in $\mathbf{Z}$. The proof of the following proposition is outlined in Appendix B.

Proposition 2.3. If $(X, \mathbf{Y}, \mathbf{Z})$ has a $(2 \mathrm{p}+1)$ EC distribution satisfying (i) $\operatorname{Cov}\left(X, Y_{i}\right)=\operatorname{Cov}\left(X, Y_{j}\right)$, (ii) $\operatorname{Cov}\left(X, Z_{i}\right)=\operatorname{Cov}\left(X, Z_{j}\right), i, j=1, \ldots, p$, then the marginal joint distribution of $\left(X, \mathbf{Y}_{\delta}, \mathbf{Z}_{\delta}\right)$ is an even function of $X-\mu_{0}$.

The following theorem describes the covariance of a covariate with the order statistics and their concomitants.

Proposition 2.4. If the distribution of $(X, \mathbf{Y}, \mathbf{Z})$ is nondegenerate elliptically contoured with arbitrary mean and arbitrary variance-covariance structure satisfying (i) and (ii) of Proposition $2.3, \operatorname{then} \operatorname{Cov}(X, \mathcal{Z})=$ $\operatorname{Cov}(X, \mathbf{Z})$.

Proof. Replacing Y by Z in expressions (2.3) and (2.4), and observing that the event $E_{p}$ is invariant under the linear transformation $(X, \mathbf{Y}) \rightarrow V=\left(X, \mathbf{Y}_{\delta}, Y_{p}\right)$, it is sufficient to show that $\operatorname{Cov}\left(X, Z_{r}-Z_{j} \mid E_{j}\right)=$ $0, j=1, \ldots, k$. Letting $j=p, s_{\delta r}=z_{r}-z_{p}$ and $\mu_{\delta r}=\mu_{2 r}-\mu_{2 p}$, from Proposition 2.3 it follows that

$$
\operatorname{Cov}\left(X, Z_{r}-Z_{p} \mid E_{p}\right)=\frac{1}{\operatorname{Pr}\left(E_{p}\right)} \int_{X \times E_{p}}\left(x-\mu_{0}\right)\left(s_{\delta r}-\mu_{\delta r}\right) f(x, \mathbf{s}) d x d \mathbf{s} .
$$

Because $f(x, \mathbf{s})$ is an even function of $\left(x-\mu_{0}\right)$, it follows that $\operatorname{Cov}\left(X, Z_{r}-Z_{p} \mid E_{p}\right)=0$. 
If $X, \mathbf{Y}, \mathbf{Z}$ are jointly multivariate normal, then $\mathbf{S}^{\prime}=\left(\mathbf{Y}_{\delta}, \mathbf{Z}_{\delta}\right)$ and $X$ are independent because their covariance terms are zero. Thus, the integral in expression (2.5) is

$$
\int_{\mathbf{X}}\left(x-\mu_{x}\right) f(x)\left[\int_{E_{p}}\left[\left(z_{r}-z_{p}\right)-\left(\mu_{2 r}-\mu_{2 p}\right)\right] f(x, \mathbf{s}) d \mathbf{s}\right] d x .
$$

The inside integral can be factored out so that the whole integral will be zero. In particular, then, if the distribution of $X, \mathbf{Y}, \mathbf{Z}$ is multivariate normal and permutation-symmetric in $\mathbf{Y}$ and $\mathbf{Z}$, then $\operatorname{Cov}(X, \mathcal{Y})=$ $\operatorname{Cov}(X, \mathbf{Y})$ and $\operatorname{Cov}(X, \mathcal{Z})=\operatorname{Cov}(X, \mathbf{Z})$.

\section{Correlation Analysis}

From Section 2, when the distribution of $(X, \mathbf{Y}, \mathbf{Z})$ is multivariate normal with block-permutation symmetry, the covariance structure of $(X, \mathcal{Y}, \mathcal{Z})$ is given by

$$
\Psi=\left[\begin{array}{lll}
\Psi_{00} & \Psi_{01} & \Psi_{02} \\
& \Psi_{11} & \Psi_{12} \\
& & \Psi_{22}
\end{array}\right],
$$

where $\left.\Psi_{00}=\sigma_{0}^{2}, \Psi_{0 i}=\sigma_{0} \sigma_{i} \gamma_{0 i} \mathbf{e}^{\prime}, \Psi_{11}=\sigma_{1}^{2}\left[\gamma_{11} \mathbf{e e}^{\prime}+\left(1-\gamma_{11}\right) \mathcal{C}\right)\right]$,

$$
\left.\Psi_{12}=\sigma_{1} \sigma_{2}\left[\gamma_{12} \mathbf{e e}^{\prime}+\left(\lambda_{12}-\gamma_{12}\right) \mathcal{C}\right)\right], \text { and } \quad \Psi_{22}=\Sigma_{22}+\frac{\sigma_{2}^{2}\left(\lambda_{12}-\gamma_{12}\right)^{2}}{\left(1-\gamma_{11}\right)}(\mathcal{C}-\mathbf{I}) .
$$

In what follows we consider bivariate correlation and regression parameters. The results extend with minor modifications when $p>2$ (fix $\mathrm{i}, \mathrm{j}=1,2$ in what follows).

3.1. Linear regression of $\mathcal{Z}$ on $\mathbf{X}$. The correlations $\eta_{1}$ between $X$ and $Z_{[i]}$ are equal,

$$
\eta_{1}=\operatorname{Corr}\left(X, Z_{[i]}\right)=\frac{\gamma_{02}}{\sqrt{1+\frac{\left(\lambda_{12}-\gamma_{12}\right)^{2}}{\left(1-\gamma_{11}\right)}\left(c_{22}-1\right)}},
$$

whereas the correlation $\eta_{2}$ between $Z_{[1]}$ and $Z_{[2]}$ is given by

$$
\eta_{2}=\operatorname{Corr}\left(Z_{[1]}, Z_{[2]}\right)=\frac{\left(1-\gamma_{11}\right) \gamma_{22}+\left(\lambda_{12}-\gamma_{12}\right)^{2} c_{12}}{\left(1-\gamma_{11}\right)+\left(\lambda_{12}-\gamma_{12}\right)^{2}\left(c_{22}-1\right)}
$$

with (e.g., Beyer (1991, p.243))

$$
\mathcal{C}=\left[\begin{array}{ll}
c_{11} & c_{12} \\
c_{21} & c_{22}
\end{array}\right]=\left[\begin{array}{ll}
0.6817 & 0.3183 \\
0.3183 & 0.6817
\end{array}\right] .
$$

When $\lambda_{12}=\gamma_{12}$, it follows that $\eta_{2}=\gamma_{22}$ and hence $\operatorname{Corr}\left(Z_{[1]}, Z_{[2]}\right)=\operatorname{Corr}\left(Z_{1}, Z_{2}\right)$. The partial correlation of $Z_{[1]}$ and $Z_{[2]}$ given $X$ is

$$
\eta_{2 \mid 0}=\frac{\left(1-\gamma_{11}\right)\left(\gamma_{22}-\gamma_{02}^{2}\right)+\left(\lambda_{12}-\gamma_{12}\right)^{2} c_{12}}{\left(1-\gamma_{11}\right)\left(1-\gamma_{02}^{2}\right)+\left(\lambda_{12}-\gamma_{12}\right)^{2}\left(c_{22}-1\right)}
$$


which is always a contraction of the product moment correlation, regardless of the composition of the covariate. From Proposition 2.4 and Corollary 2.1, the linear regression lines of each component of $\mathcal{Z}$ on $X$ are parallel, and determined by

$$
Z_{[i]}=\left(\mu_{2}-\frac{\sigma_{2} \gamma_{02}}{\sigma_{0}} \mu_{0}\right)+\frac{\sigma_{2}\left(\lambda_{12}-\gamma_{12}\right)}{\sqrt{1-\gamma_{11}}} c_{i}+\frac{\sigma_{2} \gamma_{02}}{\sigma_{0}} X
$$

where $c_{1}=-0.56419=-c_{2}$ (e.g., Beyer (p.241), 1991). Since the slope of $\mathcal{Z}$ on $X$ coincides with the slope of $\mathbf{Z}$ on $X$, the only difference is from the intercept whose distance results from the expected mean of order statistics of standard normal distribution. The corresponding mean squared error matrix can be expressed as

$$
\Psi_{22 \mid 0}=\Sigma_{22}+\frac{\sigma_{2}^{2}\left(\lambda_{12}-\gamma_{12}\right)^{2}}{\left(1-\gamma_{11}\right)}(\mathcal{C}-\mathbf{I})-\sigma_{2}^{2} \gamma_{02}^{2} \mathbf{e e}^{\prime}=\Sigma_{22 \mid 0}+\frac{\sigma_{2}^{2}\left(\lambda_{12}-\gamma_{12}\right)^{2}}{\left(1-\gamma_{11}\right)}(\mathcal{C}-\mathbf{I})
$$

In addition, for each component $Z_{[i]}$,

$$
\operatorname{MSE}\left(Z_{[i]} \mid X\right)=\operatorname{MSE}\left(Z_{i} \mid X\right)+\frac{\sigma_{2}^{2}\left(\lambda_{12}-\gamma_{12}\right)^{2}}{\left(1-\gamma_{11}\right)}\left(c_{i i}-1\right),
$$

where $c_{i i}$ is a diagonal element of $\mathcal{C}$. Since $c_{i i}<1, \operatorname{MSE}\left(Z_{[i]} \mid X\right)<\operatorname{MSE}\left(Z_{i} \mid X\right)$. The amount of improvement (or decrease in MSE) depends on $\operatorname{Cov}\left(Y_{i}, Z_{i}\right)$ and the covariance structure of $\mathbf{Y}$. For example, for fixed $\sigma_{1}^{2}$ and $\gamma_{11}$, the higher $Z_{i}$ is correlated with $Y_{i}$ relative to $Y_{j}, j \neq i$, the smaller the MSE of $Z_{[i]}$ on $X$ is. The multiple correlation coefficient is equal to $\eta_{1}^{2}$ which is the squared correlation between $Z_{[i]}$ on $X$. The multiple linear regression equation of one concomitant variable on $X$ and the other concomitant variable is

$$
Z_{[i]}=\beta_{0}+\beta_{1} X+\beta_{2} Z_{[j]}
$$

where $\beta_{2}$ is the partial correlation $\eta_{2 \mid 0}$,

$$
\beta_{1}=\frac{\gamma_{02} \sigma_{2}\left[\left(1-\gamma_{11}\right)\left(1-\gamma_{22}\right)-2 c_{12}\left(\lambda_{12}-\gamma_{12}\right)^{2}\right]}{\sigma_{0}\left[\left(1-\gamma_{11}\right)\left(1-\gamma_{02}^{2}\right)-c_{12}\left(\lambda_{12}-\gamma_{12}\right)^{2}\right]}
$$

and the intercept is given by $\left(c_{1}=-0.56419=-c_{2}\right)$

$$
\beta_{0}=E\left(Z_{[i]}\right)-\beta_{2} E\left(Z_{[j]}\right)-\beta_{1} \mu_{0}=\mu_{2}\left(1-\beta_{2}\right)-\beta_{1} \mu_{0}+\frac{\sigma_{2}\left(\lambda_{12}-\gamma_{12}\right)}{\sqrt{1-\gamma_{11}}}\left(c_{i}-\beta_{2} c_{j}\right) .
$$

3.2. Linear regression of $\mathbf{X}$ on $\mathcal{Z}$. Olkin and Viana (1995) showed that the linear regression of $X$ on $\mathbf{Y}$ and of $X$ on $\mathcal{Y}$ coincide. In Appendix $\mathrm{C}$ we show that a similar result holds for concomitants. That is, the linear regression of $X$ on $\mathcal{Z}$ is coincident with that of $X$ on $\mathbf{Z}$. However, this is not true for a simple regression of $X$ on each $Z_{[i]}$. For example, the regression line is determined by

$$
X=\mu_{0}-\beta_{1} \mu_{2}+\frac{\sigma_{2}\left(\lambda_{12}-\gamma_{12}\right)}{\sqrt{1-\gamma_{11}}} c_{i}+\beta_{1} Z_{[i]},
$$

where

$$
\beta_{1}=\frac{\gamma_{02} \sigma_{0}}{\sigma_{2}\left[1+\frac{\left(\lambda_{12}-\gamma_{12}\right)^{2}}{\left(1-\gamma_{11}\right)}\left(c_{22}-1\right)\right]}
$$




\section{A Numerical Example}

The following partial data are from a pilot study in which a number of physiological parameters were measured in a specific site on the left and right brain hemispheres of subjects participating in a study conducted at the University of Illinois at Chicago Sleep Center and General Clinical Research Center. In this example, we consider the following variables, jointly observed in a sample of approximately $N=30$ subjects:

(1) X: age;

(2) $Y_{s}$ : tissue oxygenation on the left site;

(3) $Y_{d}$ : tissue oxygenation on the right site;

(4) $Z_{s}$ : total hemoglobin on the left site;

(5) $Z_{d}$ : total hemoglobin on the right site.

Following the notation introduced earlier, we write $\mathbf{Y}^{\prime}=\left(Y_{s}, Y_{d}\right), \mathbf{Z}^{\prime}=\left(Z_{s}, Z_{d}\right)$, so that $\mathcal{Y}$ is the vector of ordered oxygenation levels, $Z_{[1]}$ is the total hemoglobin in the hemisphere with the lowest oxygenation level and $Z_{[2]}$ is the total hemoglobin in the hemisphere with the highest oxygenation level. We are interested in the linear prediction of the mean total hemoglobin in the site with extreme (lowest, highest) oxygenation level, as a function of the subject's age. This age-corrected information may be used as a potential outcome in future comparative studies.

Matrix (4.1) shows the joint sample covariance matrix

$$
S=\left[\begin{array}{c|cc|cc}
128.414 & -23.488 & -32.733 & -30.531 & -41.460 \\
\hline-23.488 & 33.283 & 26.090 & 52.281 & 41.596 \\
-32.733 & 26.090 & 46.747 & 29.261 & 48.501 \\
\hline-30.531 & 52.281 & 29.261 & 145.672 & 113.061 \\
-41.460 & 41.596 & 48.501 & 113.061 & 173.492
\end{array}\right]
$$

for $(X, \mathbf{Y}, \mathbf{Z})$, with corresponding block covariance matrices $S_{i j}$. Under the assumption that the joint distribution of $(X, \mathbf{Y}, \mathbf{Z})$ is multivariate normal $(\boldsymbol{\mu}, \Sigma)$ satisfying the block permutation-symmetry property, the MLE $\hat{\Sigma}$ of $\Sigma$ is given by $\hat{\sigma}_{0}^{2}=S_{00}, \hat{\sigma}_{i}^{2}=\operatorname{tr}\left(\mathbf{S}_{i i}\right) / p, \hat{\lambda}_{12}=\operatorname{tr}\left(\mathbf{S}_{12}\right) / p$,

$$
\hat{\gamma}_{0 i}=\frac{\overline{\mathbf{S}}_{0 i}}{p \hat{\sigma}_{0} \hat{\sigma}_{i}}, \quad \hat{\gamma}_{i i}=\frac{\overline{\mathbf{S}}_{i i}-\operatorname{tr}\left(\mathbf{S}_{i i}\right)}{(p-1) \operatorname{tr}\left(\mathbf{S}_{i i}\right)}, \quad \hat{\gamma}_{12}=\frac{\overline{\mathbf{S}}_{12}-\operatorname{tr}\left(\mathbf{S}_{12}\right)}{p(p-1) \hat{\sigma}_{1} \hat{\sigma}_{2}},
$$


where $\bar{S}_{i j}$ indicates the sum of all entries of $S_{i j}$, that is, $\bar{S}_{i j}=\mathbf{e}^{\prime} S \mathbf{e}, i, j=1,2$. The resulting centralized sample covariance matrix is then

$$
\widehat{\Sigma}=\left[\begin{array}{c|cc|cc}
128.41 & -28.110 & -28.110 & -35.996 & -35.996 \\
\hline-28.110 & 40.016 & 26.090 & 50.390 & 35.428 \\
-28.110 & 26.090 & 40.016 & 35.428 & 50.390 \\
\hline-35.996 & 50.390 & 35.428 & 159.58 & 113.06 \\
-35.996 & 35.428 & 50.390 & 113.06 & 159.58
\end{array}\right]
$$

From Section 3, the components of the estimated covariance structure $\widehat{\Psi}$ of $(X, \mathcal{Y}, \mathcal{Z})$ are given by

$$
\widehat{\Psi}_{0 i}=\widehat{\Sigma}_{0 i}, \quad i=0,1,2, \quad \widehat{\Psi}_{11}=\widehat{\Sigma}_{11} \mathcal{C}=\left[\begin{array}{cc}
35.574 & 30.532 \\
30.532 & 35.574
\end{array}\right]
$$

where $\mathcal{C}$ is the covariance structure for two ordered independent standard normal random variables, shown in expression (3.4),

$$
\widehat{\Psi}_{12}=\widehat{\Sigma}_{12} \mathcal{C}=\left[\begin{array}{cc}
45.618 & 40.200 \\
40.200 & 45.618
\end{array}\right], \quad \widehat{\Psi}_{22}=\widehat{\Sigma}_{22}+T\left(\mathcal{C}-\widehat{\Sigma}_{11}\right) T=\left[\begin{array}{cc}
159.21 & 113.43 \\
113.43 & 159.21
\end{array}\right],
$$

where

$$
T=\widehat{\Sigma}_{12} \widehat{\Sigma}_{11}^{-1}=\left[\begin{array}{cc}
1.1863 & 0.1118 \\
0.1118 & 1.1863
\end{array}\right]
$$

In summary,

$$
\widehat{\operatorname{Cov}}[(X, \mathcal{Y}, \mathcal{Z})]=\left[\begin{array}{c|ccc|cc}
128.41 & -28.110 & -28.110 & -35.996 & -35.996 \\
\hline-28.110 & 35.574 & 30.532 & 45.618 & 40.200 \\
-28.110 & 30.532 & 35.574 & 40.200 & 45.618 \\
\hline-35.996 & 45.618 & 40.200 & 159.21 & 113.43 \\
-35.996 & 40.200 & 45.618 & 113.43 & 159.21
\end{array}\right] .
$$

The estimated linear regression of $Z_{[i]}$ on $X$ follows from Section expression (3.6) in Section 3.1. We obtained

$$
Z_{[1]}=51.362-0.287 X, \quad Z_{[2]}=55.908-0.287 X .
$$




\section{Discussion and Complementary Results}

We have considered the ordered components, $\mathcal{Y}$, of a multivariate random variable, $\mathbf{Y}$, with covariance matrix $\Sigma_{11}$ and the vector, $\mathcal{Z}$, of concomitantly or induced ordered components of a secondary random vector, $\mathbf{Z}$, with covariance matrix $\Sigma_{22}$. Assuming that $\Sigma_{11}, \Sigma_{22}$ and the covariance structure between $\mathbf{Y}$ and $\mathbf{Z}$ are permutation-symmetric, the joint covariance structure for $\mathcal{Y}$ and $\mathcal{Z}$ was obtained. The case in which the joint probability distribution of $(\mathbf{Y}, \mathbf{Z})$ is multivariate normal leads to an explicit formulation of the covariances of interest.

5.1. Variance of the estimates. Large-sample estimates of the variance of the estimated covariance structure $\widehat{\Sigma}$ of $(X, \mathbf{Y}, \mathbf{Z})$ follow from the fact that $\sqrt{N}(\hat{\Sigma}-\Sigma) \stackrel{\mathcal{L}}{\rightarrow} \mathbf{N}(\mathbf{0}, \mathcal{A} \operatorname{Cov}(\Sigma))$, where the entries of the asymptotic covariance matrix $\mathcal{A} \operatorname{Cov}(\Sigma)$ of $\Sigma$ follow from the standard delta method. For example, when $\mathrm{p}=2$,

$$
\mathcal{A} \operatorname{Cov}\left(\lambda_{12}, \gamma_{12}\right)=\left[\begin{array}{ll}
\frac{1}{2}\left(1+\lambda_{12}^{2}+\gamma_{12}^{2}+\gamma_{11} \gamma_{22}\right) & \lambda_{12} \gamma_{12}+\frac{1}{2}\left(\gamma_{11}+\gamma_{22}\right) \\
\lambda_{12} \gamma_{12}+\frac{1}{2}\left(\gamma_{11}+\gamma_{22}\right) & \frac{1}{2}\left(1+\lambda_{12}^{2}+\gamma_{12}^{2}+\gamma_{11} \gamma_{22}\right)
\end{array}\right]
$$

can be used to assess the hypothesis $\lambda_{12}=\gamma_{12}$. The closed-form large-sample covariance of the components of $\hat{\Sigma}$ for $p=2$ is reported in Lee (1998). See also Viana and Olkin (2000) and Viana and Olkin (1998) for additional results on symmetrically dependent models.

5.2. Siegel's Argument. Siegel (1993) showed that when $\left(X_{1}, \ldots, X_{n}\right)$ is multivariate normal with arbitrary mean and covariance structure, then $\operatorname{Cov}\left(X_{1}, X_{(1)}\right)=\sum_{i=1}^{n} \operatorname{Cov}\left(X_{1}, X_{i}\right) \operatorname{Pr}\left(X_{i}=X_{(1)}\right)$. The result holds, surprisingly, even when $X_{1}$, say, is not ordered among the remaining $p=n-1$ components (indicate here by $\left.Y_{1}, \ldots, Y_{p}\right)$, that is, $\operatorname{Cov}\left(X_{1}, Y_{(1)}\right)=\sum_{i=2}^{n} \operatorname{Cov}\left(X_{1}, X_{i}\right) \operatorname{Pr}\left(X_{i}=Y_{(1)}\right)$. Therefore, when $X_{1}$ is equicorrelated with the remaining components, one obtains $\operatorname{Cov}\left(X_{1}, Y_{(1)}\right)=\operatorname{Cov}\left(X_{1}, Y_{i}\right), i=1 \ldots p$. This is later extended by Rinott and Samuel-Cahn (1994) to an arbitrary order statistics, and hence, in the present notation, $\operatorname{Cov}(X, \mathcal{Y})=\operatorname{Cov}(X, Y)$. Liu (1994) showed that Siegel's result follows from a multivariate version of Stein's identity (Stein (1981)) and Anderson (1993) showed, in a short and elegant argument, that there is a relationship between Siegel's proof and the classic identity of Stein.

5.3. David's representation. David (1996) suggested a representation of equally correlated variates due to C. Thigpen (see David, 1981, p.117) which leads to the covariance structure of order statistics. If $(X, \mathbf{Y})$ is permutation symmetric normal with $\operatorname{Cov}\left(Y_{i}, Y_{j}\right)=\gamma_{11}, i \neq j, i, j=1, \ldots, p$ and $\operatorname{Cov}\left(X, Y_{i}\right)=\gamma_{01}, i=$ $1, \ldots, p$, then $Y_{i}$ can be represented as $Y_{i}=\gamma_{01} X+\sqrt{1-\gamma_{01}^{2}} V_{i}, i=1, \ldots, p$, where the $V_{i}$ are independent of $X$ and standard normals with a common correlation coefficient $\left(\gamma_{11}-\gamma_{01}^{2}\right) /\left(1-\gamma_{01}^{2}\right)$. In this case, $\mathcal{Y}=\gamma_{01} X+\sqrt{1-\gamma_{01}^{2}} \mathcal{V}$, where $\mathcal{V}$ is the order vector of $\mathbf{V}$. It then follows that $\operatorname{Cov}(X, \mathcal{Y})=\gamma_{01}=\operatorname{Cov}(X, \mathbf{Y})$. This representation is useful when the distribution of $\mathbf{Y}$ is permutation symmetric. David's representation can also be used to obtain the covariance of concomitants of order statistics and a covariate, when the 
additional variables, $Z_{1}, \ldots, Z_{p}$ are permutation symmetric, and the covariance matrix of $(X, \mathbf{Y}, \mathbf{Z})$ has the BPS. Assume joint normality and let $\sigma_{0}^{2}=\sigma_{1}^{2}=\sigma_{2}^{2}=1$ (without loss of generality). Then, $T_{i}^{\prime}=\left(Y_{i}, Z_{i}\right.$ ) may be represented as $T_{i}=\gamma X+\Gamma S_{i}, i=1, \ldots, p$ where

$$
S_{i}=\left[\begin{array}{c}
V_{i} \\
W_{i}
\end{array}\right], \gamma=\left[\begin{array}{c}
\gamma_{01} \\
\gamma_{02}
\end{array}\right] \text { and } \Gamma=\left[\begin{array}{ll}
\sqrt{1-\gamma_{01}^{2}} & 0 \\
0 & \sqrt{1-\gamma_{02}^{2}}
\end{array}\right] \text {. }
$$

Therefore,

$$
\operatorname{Var}\left(T_{i}\right)=\left[\begin{array}{ll}
1 & \lambda_{12} \\
\lambda_{12} & 1
\end{array}\right], \quad \operatorname{Cov}\left(T_{i}, T_{j}\right)=\left[\begin{array}{ll}
\gamma_{11} & \gamma_{12} \\
\gamma_{12} & \gamma_{22}
\end{array}\right]
$$

Moreover, $S_{i}$ is multivariate normal independent of $X$, with

$\operatorname{Cov}\left(S_{i}\right)=\left[\begin{array}{ll}1 & \frac{\lambda_{12}-\gamma_{01} \gamma_{02}}{\sqrt{1-\gamma_{01}^{2}} \sqrt{1-\gamma_{2}^{02}}} \\ \frac{\lambda_{12}-\gamma_{01} \gamma_{02}}{\sqrt{1-\gamma_{01}^{2}} \sqrt{1-\gamma_{02}^{2}}} & 1\end{array}\right], \operatorname{Cov}\left[S_{i}, S_{j}\right]=\left[\begin{array}{ll}\frac{\gamma_{11}-\gamma_{01}^{2}}{1-\gamma_{01}^{2}} & \frac{\gamma_{12}-\gamma_{01} \gamma_{02}}{\sqrt{1-\gamma_{01}^{2}} \sqrt{1-\gamma_{02}^{2}}} \\ \frac{\gamma_{12}-\gamma_{01} \gamma_{02}}{\sqrt{1-\gamma_{01}^{2}} \sqrt{1-\gamma_{02}^{2}}} & \frac{\gamma_{22}-\gamma_{02}^{2}}{1-\gamma_{02}^{2}}\end{array}\right], i \neq j$.

Define $T_{(r)}$ according to the value of the $Y_{i}$ as $T_{(r)}^{\prime}=\left(Y_{(r)}, Z_{[r]}\right)$. Since the ordering of Y-variates depends on that of V-variates, it follows that $T_{(r)}=\gamma X+\Gamma S_{(r)}$, for $r=1, \ldots, p$, where $S_{(r)}^{\prime}=\left(V_{(r)}\right.$, $\left.W_{[r]}\right)$. Consequently, for $r, i=1, \ldots, p, i=1, \ldots, p, \operatorname{Cov}\left(X, T_{(r)}\right)=\gamma=\operatorname{Cov}\left(X, T_{i}\right)$ so that $\operatorname{Cov}\left(X, Y_{(r)}\right)=\gamma_{01}=\operatorname{Cov}\left(X, Y_{i}\right)$, and $\operatorname{Cov}\left(X, Z_{[r]]}\right)=\gamma_{02}=\operatorname{Cov}\left(X, Z_{i}\right)$

5.4. A representation for order statistics, concomitants and ranks. In Section 2, we represented the concomitants of order statistics as the product $\mathbf{U Z}$ of $\mathbf{Z}$ and a random permutation matrix $\mathbf{U}$. More specifically, given any $\mathbf{Y} \in R^{p}$, there is one (uniquely defined when the components of $\mathbf{Y}$ are distinct) permutation $\tau$ ordering the components of $\mathbf{Y}$, that is,

$$
Y(\tau 1)<Y(\tau 2)<\ldots<Y(\tau p)
$$

If we indicate by $\mathbf{U}=\mathbf{U}(\tau)$ the permutation matrix defined by $\tau$, than it follows that the $\mathbf{U Y}$ is the vector of order statistics and $\mathbf{U Z}$ is its vector of induced order statistics. Moreover, if $r^{\prime}=(1,2, \ldots, p)$, then $\mathbf{U} r$ is the associated vector $R$ of ranks. That is,

$$
\mathcal{Y}=\mathbf{U Y}, \quad \mathcal{Z}=\mathbf{U Z}, \quad R=\mathbf{U} r .
$$

The set $S_{p}$ of all permutations in $p$ elements has the algebraic structure of a group and $\mathbf{U}$ is a linear representation of $S_{p}$, in the sense that $\mathbf{U}(\tau)$ are non-singular matrices satisfying $\mathbf{U}(\tau) \mathbf{U}(\sigma)=\mathbf{U}(\tau \sigma)$ for all $\tau, \sigma$ in $S_{p}$. Basic notions of group theory can then be used to facilitate the understanding of certain symmetry-related properties of order statistics and their covariance structure. e.g., Viana (2003), Viana $(2005)$.

For example, when the underlying probability model $(f)$ of $\mathbf{Y}$ is permutation-symmetric, so that $f(\mathbf{y})=$ $f(u \mathbf{y})$ for all permutation matrices $u$, then $\mathbf{U}=\mathbf{U}(\tau)$ defined by (5.1) is a random permutation matrix uniformly distributed in $S_{p}$. 
To prove Proposition 2.1, we pointed to the fact that permutation-symmetric matrices commute with every permutation matrix of corresponding dimension, that is, if $\Sigma=\alpha \mathbf{e} \mathbf{e}^{\prime}+\beta \mathbf{I}$ for real scalars $\alpha$ and $\beta$, then $u \Sigma=\Sigma u$ for all permutations $u$ of same dimension as $\Sigma$ (the converse being also true).

The case $\lambda_{12}=\gamma_{12}$ in Corollary 2.1 is of particular interest. This is the case when the covariance structure between $\mathbf{Y}$ and $\mathbf{Z}$ is proportional to $\mathbf{e e}^{\prime}$. Then we have $\operatorname{Cov}(\mathbf{Y}, \mathbf{Z})=\operatorname{Cov}(\mathcal{Y}, \mathcal{Z})$. Furthermore, $E(\mathbf{Z})=E(\mathcal{Z})$ and $\operatorname{Cov}(\mathbf{Z})=\operatorname{Cov}(\mathcal{Z})$ so that the distribution of $\mathcal{Z}$ is not affected by ordering on the $\mathbf{Y}$ space.

A related result for two symmetrically dependent ordered multivariate normal variates is as follows: Suppose that the joint distribution of $\left(\mathbf{Y}_{1}, \mathbf{Y}_{2}\right)$ is multivariate normal satisfying the BPS condition, and $\mathbf{Y}_{1}$ and $\mathbf{Y}_{2}$ are related by $\mathbf{Y}_{2}=\mathbf{T} \mathbf{Y}_{1}+\mathbf{V}$, where $\mathbf{Y}_{1}$ and $\mathbf{V}$ are independent and $\mathbf{T}=\Sigma_{21} \Sigma_{11}^{-1}$. Let $\mathcal{Y}_{i}$ indicate the ordered version of $\mathbf{Y}_{i}, \mathrm{i}=1,2$. Then

$$
\operatorname{Cov}\left[\left(\mathcal{Y}_{1}, \mathcal{Y}_{2}\right)\right]=\mathbf{A}\left[\operatorname{Cov}\left[\left(\mathbf{Y}_{1}, \mathbf{V}\right)\right] \otimes \mathcal{C}\right] \mathbf{A}^{\prime}=\mathbf{A} \operatorname{Cov}\left[\left(\mathbf{Y}_{1}, \mathbf{V}\right)\right] \mathbf{A}^{\prime} \otimes \mathcal{C}=\operatorname{Cov}\left[\left(\mathbf{Y}_{1}, \mathbf{Y}_{2}\right)\right] \otimes \mathcal{C}
$$

where $\otimes$ indicates the Kronecker product of block matrices and $\mathbf{A}=\left[\begin{array}{ll}\mathbf{I} & \mathbf{0} \\ \mathbf{T} & \mathbf{I}\end{array}\right]$. When $\lambda_{12}=\gamma_{12}$, using the fact that $\Sigma_{12} \mathcal{C}=\Sigma_{12}$, then $\operatorname{Cov}\left(\mathcal{Y}_{1}, \mathcal{Y}_{2}\right)=\operatorname{Cov}\left(\mathbf{Y}_{1}, \mathbf{Y}_{2}\right)=\Sigma_{12}$, as shown in Viana and Olkin (1997).

\section{Appendix A. Proof of Lemma 2.1}

Proof. Let $\mathbf{V}^{\prime}=\left(X, \mathbf{Y}_{\delta}, Y_{p}\right)$ so that $\mathbf{V}=\mathbf{A W}$ with

$$
\mathbf{A}=\left[\begin{array}{lll}
1 & \mathbf{0} & 0 \\
\mathbf{0} & \mathbf{I} & -\mathbf{e} \\
0 & \mathbf{0} & 1
\end{array}\right] \quad \text { and } \quad \mathbf{A}^{-1}=\left[\begin{array}{ccc}
1 & \mathbf{0} & 0 \\
\mathbf{0} & \mathbf{I} & \mathbf{e} \\
0 & \mathbf{0} & 1
\end{array}\right]
$$

The Jacobian of the transformation is $\left|\mathbf{A}^{-1}\right|=1$ and the density function of $\mathbf{V}$ is

$$
\begin{aligned}
f(\mathbf{v}) & =|\mathbf{A}|^{-\frac{1}{2}}|\Sigma|^{-\frac{1}{2}}\left|\mathbf{A}^{\prime}\right|^{-\frac{1}{2}} g\left(\left(\mathbf{A}^{-1} \mathbf{v}-\boldsymbol{\mu}\right)^{\prime} \Sigma^{-1}\left(\mathbf{A}^{-1} \mathbf{v}-\boldsymbol{\mu}\right)\right) \\
& =\left|\mathbf{A} \Sigma \mathbf{A}^{\prime}\right|^{-\frac{1}{2}} g\left(\left(\mathbf{v}-\mathbf{A}^{-1} \boldsymbol{\mu}\right)^{\prime}\left(\mathbf{A} \Sigma \mathbf{A}^{\prime}\right)^{-1}\left(\mathbf{v}-\mathbf{A}^{-1} \boldsymbol{\mu}\right)\right) \\
& =\left|\Sigma_{\mathbf{v}}\right|^{-\frac{1}{2}} g_{\mathbf{v}}\left(\left(\mathbf{v}-\boldsymbol{\mu}_{\mathbf{v}}\right)^{\prime} \Sigma_{\mathbf{v}}^{-1}\left(\mathbf{v}-\boldsymbol{\mu}_{\mathbf{v}}\right)\right),
\end{aligned}
$$

where $\boldsymbol{\mu}_{\mathbf{v}}^{\prime}=\left(\mu_{0}, \mu_{1}-\mu_{p}, \ldots, \mu_{p-1}-\mu_{p}, \mu_{p}\right)$, and

$$
\Sigma_{\mathbf{v}}=\mathbf{A} \Sigma \mathbf{A}^{\prime}=\left[\begin{array}{ccc}
\sigma_{0}^{2} & 0 \mathbf{e}^{\prime} & \alpha \\
0 \mathbf{e} & \Sigma_{\delta} & \Sigma_{\delta p} \\
\alpha & \Sigma_{p d} & \sigma_{p}^{2}
\end{array}\right]
$$

Here $\sigma_{p}^{2}$ is a component for $Y_{p}$ in $\Sigma, \Sigma_{\delta}$ is a $(p-1) \times(p-1)$ matrix associated with $\operatorname{Cov}\left(\mathbf{Y}_{\delta}\right)$, and $\Sigma_{\delta p}$ is associated with $\operatorname{Cov}\left(\mathbf{Y}_{\delta}, Y_{p}\right)$. From this transformation, the distribution of $\mathbf{V}$ is $\mathbf{E C}$ so that the marginal 
distribution for $\left(X, \mathbf{Y}_{\delta}\right)$ is also EC. Its density is given by

$$
f\left(x, \mathbf{y}_{\delta}\right)=\left(\sigma_{0}^{-1}\right)\left|\Sigma_{\delta}\right|^{\frac{-1}{2}} g\left(\frac{\left(x-\mu_{0}\right)^{2}}{\sigma_{0}^{2}}+\left(\mathbf{y}_{\delta}-\boldsymbol{\mu}_{\delta}\right)^{\prime} \Sigma_{\delta}^{-1}\left(\mathbf{y}_{\delta}-\boldsymbol{\mu}_{\delta}\right)\right),
$$

where $\boldsymbol{\mu}_{\delta}^{\prime}=\left(\mu_{1}-\mu_{p}, \ldots, \mu_{p-1}-\mu_{p}\right)$. The first component in the function $g$ is an even function of $\left(X-\mu_{0}\right)$, and the second part is constant in $x$, concluding the proof.

\section{Appendix B. Proof of Proposition 2.3}

Proof. Let $\mathbf{S}^{\prime}=\left(\mathbf{Y}_{\delta}, \mathbf{Z}_{\delta}\right)$ and $\mathbf{V}^{\prime}=\left(X, \mathbf{S}^{\prime}, Y_{p}, Z_{p}\right)$. Then $\mathbf{V}=\mathbf{A W}$, with $\boldsymbol{\mu}_{\mathbf{s}}^{\prime}=\left(\mu_{11}-\mu_{1 p}, \ldots, \mu_{1(p-1)}, \mu_{21}-\right.$ $\left.\mu_{2 p}, \ldots, \mu_{2(p-1)}-\mu_{2 p}\right)$ and

$$
\mathbf{A}=\left[\begin{array}{lll}
1 & \mathbf{0} & \mathbf{0} \\
\mathbf{0} & \mathbf{I} & -\mathbf{e} \otimes \mathbf{I} \\
\mathbf{0} & \mathbf{0} & \mathbf{I}
\end{array}\right] .
$$

The Jacobian of the transformation from $\mathbf{W}$ to $\mathbf{V}$ is $|\mathbf{J}|=\left|\mathbf{A}^{-1}\right|=1$. The density of $\mathbf{V}$ is $f(\mathbf{v})=$ $\left|\Sigma_{\mathbf{v}}\right|^{\frac{-1}{2}} g_{\mathbf{v}}\left(\left(\mathbf{v}-\boldsymbol{\mu}_{\mathbf{v}}\right)^{\prime} \Sigma_{\mathbf{v}}^{-1}\left(\mathbf{v}-\boldsymbol{\mu}_{\mathbf{v}}\right)\right)$, where $\boldsymbol{\mu}_{\mathbf{v}}^{\prime}=\left(\mu_{0}, \boldsymbol{\mu}_{\mathbf{s}}^{\prime}, \mu_{1 p}, \mu_{2 p}\right)$ and

$$
\Sigma_{\mathbf{v}}=\left[\begin{array}{lll}
\sigma_{0}^{2} & \mathbf{0} & (\alpha, \beta) \\
\mathbf{0} & \Sigma_{\mathbf{s}} & \Sigma_{3} \\
(\alpha, \beta)^{\prime} & \Sigma_{3} & \sigma_{p}^{2}
\end{array}\right] .
$$

Here $\Sigma_{\mathbf{s}}$ is a $2(p-1) \times 2(p-1)$ matrix associated with $\operatorname{Cov}\left(\mathbf{Y}_{\delta}, \mathbf{Z}_{\delta}\right)$ and $\Sigma_{3}$ is a $2(p-1) \times 2$ matrix associated with $\operatorname{Cov}\left(\left(\mathbf{Y}_{\delta}, \mathbf{Z}_{\delta}\right),\left(Y_{p}, Z_{p}\right)\right)$. The marginal distribution of $(X, \mathbf{S})$ is also $\mathbf{E C}$ and its density is

$$
f(x, \mathbf{s})=\left(\sigma_{0}^{-1}\right)\left|\Sigma_{\mathbf{s}}\right|^{\frac{-1}{2}} g\left[\frac{\left(x-\mu_{0}\right)^{2}}{\sigma_{0}^{2}}+\left(\mathbf{s}-\boldsymbol{\mu}_{\mathbf{s}}\right)^{\prime} \Sigma_{\mathbf{s}}^{-1}\left(\mathbf{s}-\boldsymbol{\mu}_{\mathbf{s}}\right)\right],
$$

showing the function $g$ is an even function of $\left(x-\mu_{0}\right)$.

\section{Appendix C. Linear Regression of X ON $\mathcal{Z}$}

The linear regression of $X$ on $\mathcal{Z}$ is coincident with that of $X$ on $\mathbf{Z}$. To see this, start with the best linear predictor of $X$ on $\mathcal{Z}, \mu_{0}+\Psi_{02} \Psi_{22}^{-1}[\mathcal{Z}-E(\mathcal{Z})]$, where

$$
\begin{aligned}
\Psi_{02} \Psi_{22}^{-1} & =\sigma_{0} \sigma_{2} \gamma_{02} \mathbf{e}^{\prime}\left[\Sigma_{22}+\frac{\sigma_{2}^{2}\left(\lambda_{12}-\gamma_{12}\right)^{2}}{\left(1-\gamma_{11}\right)}(\mathcal{C}-\mathbf{I})\right]^{-1} \\
& =\sigma_{0} \sigma_{2} \gamma_{02} \mathbf{e}^{\prime} \Sigma_{22}^{-1}\left[\mathbf{I}+\frac{\sigma_{2}^{2}\left(\lambda_{12}-\gamma_{12}\right)^{2}}{\left(1-\gamma_{11}\right)}(\mathcal{C}-\mathbf{I}) \Sigma_{22}^{-1}\right]^{-1} .
\end{aligned}
$$

Because $\Sigma_{22}^{-1}=1 /\left[\sigma_{2}^{2}\left(1-\gamma_{22}\right)\right]$, and

$\mathbf{e}^{\prime} \Sigma_{22}^{-1}=\mathbf{e}^{\prime} \frac{1}{\sigma_{2}^{2}\left(1-\gamma_{22}\right)}\left[\mathbf{I}-\frac{\gamma_{22}}{1+\gamma_{22}(p-1)} \mathbf{e e}^{\prime}\right]=\frac{1}{\sigma_{2}^{2}\left(1-\gamma_{22}\right)}\left(1-\frac{p \gamma_{22}}{1+\gamma_{22}(p-1)}\right) \mathbf{e}^{\prime}=\frac{1}{\sigma_{2}^{2}\left(1+\gamma_{22}(p-1)\right)} \mathbf{e}^{\prime}$, 
it follows that

$$
\begin{aligned}
\Psi_{02} \Psi_{22}^{-1} & =\frac{\gamma_{02} \sigma_{0}}{\sigma_{2}\left(1+\gamma_{22}(p-1)\right)} \mathbf{e}^{\prime}\left[\mathbf{I}+\frac{\left(\lambda_{12}-\gamma_{12}\right)^{2}}{\left(1-\gamma_{11}\right)\left(1-\gamma_{22}\right)}(\mathcal{C}-\mathbf{I})\right]^{-1} \\
& =\frac{\gamma_{02} \sigma_{0}}{\sigma_{2}\left(1+\gamma_{22}(p-1)\right)} \mathbf{e}^{\prime}((1-\psi) \mathbf{I}+\psi \mathcal{C})^{-1}
\end{aligned}
$$

with $\psi=\left(\lambda_{12}-\gamma_{12}\right)^{2} /\left[\left(1-\gamma_{11}\right)\left(1-\gamma_{22}\right)\right]$. Moreover, $\mathbf{e}^{\prime} \mathcal{C}=\mathbf{e}^{\prime}$, so that

$$
\Psi_{02} \Psi_{22}^{-1}=\frac{\gamma_{02} \sigma_{0}}{\sigma_{2}\left(1+\gamma_{22}(p-1)\right)} \mathbf{e}^{\prime}=\Sigma_{02} \Sigma_{22}^{-1} .
$$

Finally, because $\mathbf{e}^{\prime} \mathbf{c}=0$,

$$
\begin{aligned}
\text { intercept } & =\mu_{0}-\Psi_{02} \Psi_{22}^{-1} E(\mathcal{Z})=\mu_{0}-\frac{\gamma_{02} \sigma_{0}}{\sigma_{2}\left(1+\gamma_{22}(p-1)\right)} \mathbf{e}^{\prime}\left[\mu_{2} \mathbf{e}+\frac{\sigma_{2}}{\sqrt{1-\gamma_{11}}}\left(\lambda_{12}-\gamma_{12}\right) \mathbf{c}\right] \\
& =\mu_{0}-\frac{p \gamma_{02} \sigma_{0}}{\sigma_{2}\left(1+\gamma_{22}(p-1)\right)} \mu_{2},
\end{aligned}
$$

which is the intercept of the regression of $X$ on $\mathbf{Z}$. Therefore, the two regression equations, $X$ on $\mathbf{Z}$ and $X$ on $\mathcal{Z}$, are the same.

The University of Illinois at Chicago

College of Medicine

1855 West Taylor Street, M/C 648

Chicago Illinois 60612

Abbott Laboratories

ImmunoScience Development Center

300 Interpace Parkway

Parsippany, NJ 07054 


\section{REFERENCES}

Anderson, C. L. (1993), 'Extension of surprising covariance', Journal of the American Statistical Association 88, 1478.

Beyer, W. H. (1991), Standard Probability and Statistics Tables and Formulae, CRC Press, Boca Raton.

Bhattacharya, P. K. (1976), 'An invariance principle in regression analysis', Annals of Statististics 4, 621-624.

Bhattacharya, P. K. (1984), Induced order statistics: theory and application, in P. K. Rishnaiah and P. K. Sen, eds, 'Handbook of Statistics', Vol. 4, pp. 383-403.

David, H. A. (1981), Order Statistics, Wiley, New York.

David, H. A. (1996), 'A general representation of equally correlated variates', Journal of the American Statistical Association 91(436), 1756.

David, H. A. and Galambos, J. (1974), 'The asymptotic theory of concomitants of order statistics', Journal of Applied Probability 11, $762-770$.

David, H. A. and Nagaraja, H. N. (1998), Concomitants of order statistics., in N. Balakrishnan and C. R. Rao, eds, 'Handbook of Statistics', Vol. 16, North-Holland, New York, NY, chapter 18, pp. 487-513.

Lee, H. (1998), The covariance structure of concomitants of ordered symmetrically dependent observations, PhD thesis, The University of Illinois at Chicago Division of Biostatistics.

Lee, H. and Viana, M. (1999), 'The joint covariance structure of ordered symmetrically dependent observations and their concomitants of order statistics', Statistics and Probability Letters 43, 411-414.

Liu, J. S. (1994), 'Siegel's formula via stein's identities', Statistics and Probability Letters 21, 247-251.

Olkin, I. and Viana, M. (1995), 'Correlation analysis of extreme observations from a multivariate normal distribution.', Journal of the American Statistical Association 90, 1373-1379.

Owen, D. B. and Steck, G. P. (1962), 'Moments of order statistics from the equicorrelated multivariate normal distribution', Annals of Mathematical Statistics 33, 1286-1291.

Rinott, Y. and Samuel-Cahn, E. (1994), 'Covariance between variables and their order statistics for multivariate normal variables', Statistics and Probability Letters 21, 153-155.

Siegel, A. F. (1993), 'A surprising covariance involving the minimum of multivariate normal variables.', J. Amer. Statist. Assn. 88, $77-80$.

Stein, C. M. (1981), 'Estimation of the mean of a multivariate normal distribution.', Ann. Statist. 6, 1135-1151.

Viana, M. (2003), Symmetry Studies- An Introduction, IMPA Institute for Pure and Applied Mathematcis Press, Rio de Janeiro, Brazil.

Viana, M. (2005), Lecture Notes on Symmetry Studies, EURANDOM, Technische Universiteit Eindhoven, Eindhoven, NL.

Viana, M. A. G. (1998), Linear combinations of ordered symmetric observations with applications to visual acuity, in N. Balakrishnan and C. R. Rao, eds, 'Order Statistics: Applications', Vol. 17, Elsevier, Amsterdam, chapter 19, pp. 513-24.

Viana, M. A. G., Olkin, I. and McMahon, T. (1993), 'Multivariate assessment of computer analyzed corneal topographers', Journal of the Optical Society of America - A 10(8), 1826-1834.

Viana, M. and Olkin, I. (1997), Correlation analysis of ordered observations from a block-equicorrelated multivariate normal distribution, in S. Panchapakesan and N. Balakrishnan, eds, 'Advances in Statistical Decision Theory and Applications', Birkhauser, Boston, MA, chapter 21.

Viana, M. and Olkin, I. (1998), Symmetrically dependent models arising in visual assessment data, Technical Report 1998-11, Statistics Department, Stanford University.

Viana, M. and Olkin, I. (2000), 'Symmetrically dependent models arising in visual assessment data', Biometrics (56), $1188-1191$. 